\title{
EXAMPLES OF MALFORMED SUBSETS OF A RIEMANN SURFACE
}

\author{
by MOSES GLASNER
}

(Received 14 April, 1981)

Let $R$ be a hyperbolic Riemann surface and $W$ an open subset of $R$ with $\partial W$ piecewise analytic. Denote by $\tilde{M}(R)$ the space of Dirichlet finite Tonelli functions on $R$ and by $\pi$ the harmonic projection of $\tilde{M}(R)$. Consider the relative $H D$-class on $W, H D(W ; \partial W)=\{u \in \tilde{M}(R)|u| W \in H D(W)$ and $u \mid R \backslash W=0\}$. The extremization operation $\mu_{D}^{W}$ is the linear mapping of $H D(W ; \partial W)$ into $H D(R)$ defined by $\mu_{D}^{W}=$ $\pi \mid H D(W ; \partial W)$. Since $\pi$ preserves values of functions at the Royden harmonic boundary, the maximum principle implies that $\mu_{D}^{W}$ is an order preserving injection and that $\mu_{B D}^{W}=\mu_{D}^{W} \mid H B D(W ; \partial W)$ is an isometry with respect to the supremum norms.

The term malformed was introduced by Nakai [2] for subsets $W$ of $R$ with the propery that $\mu_{B D}^{w}$ is surjective yet $\mu_{D}^{W}$ is not. Royden's theorem [4] on the $C D$-denseness of $H B D(R)$ in $H D(R)$ (and that of $H B D(W ; \partial W)$ in $H D(W ; \partial W)$ ) leads one to believe that there are no malformed subsets. However, Nakai $[1,2]$ showed that on an arbitrary hyperbolic Riemann surface $R$ corresponding to each unbounded function $h \in H D(R)$ there is a malformed region $W$ with $h \notin \mu_{D}^{W}(H D(W ; \partial W))$. The proof of this important result on the classification of Riemann surfaces is elaborate and difficult. In this paper we show that on a suitably chosen $R$ the task of exhibiting malformed subregions is considerably simpler. From this point of view the result presented here is weaker. However, due to its simplicity one can observe a new phenomenon: the existence of malformed subregions such that the deficiency of $\mu_{D}^{W}$, i.e. the dimension of $H D(R) / \mu_{D}^{W}(H D(W ; \partial W))$, is infinite.

1. We use the Royden ideal boundary extensively and refer to [5] for the notations and results. Let $T$ be the Tôki surface, a Riemann surface in the class $O_{H D} \backslash O_{G}$. The harmonic boundary of $T$ consists of a single point $p^{*}$. Thus, if $U$ is any subregion of $T$ with $T \backslash U$ compact, then $\operatorname{dim} H D(U ; \partial U)=\operatorname{dim} H B D(U ; \partial U)=1$. Let $g_{T}$ be the Green's function on $T$ with pole at a fixed point $q_{0} \in T$. For any $\alpha \in(0,+\infty)$ let $G_{\alpha}=$ $\left\{p \in T \mid g_{T}(p)<\alpha\right\}$ and set $\omega_{\alpha}=\left(1-\alpha^{-1} g_{T}\right) \cup 0$. Note that $\omega_{\alpha} \in H B D\left(G_{\alpha} ; \partial G_{\alpha}\right)$ and $\omega_{\alpha}\left(p^{*}\right)=1$.

Lemma. For $\alpha \in(0,+\infty)$ the Dirichlet integral of $\omega_{\alpha}$ over $T$ is given by

$$
D_{T}\left(\omega_{\alpha}\right)=2 \pi / \alpha \text {. }
$$

For the proof consider the polar coordinate differentials $d r, d \theta$ on $T$ defined by $d r / r=-d g_{T}, d \theta=-{ }^{*} d g_{T}$. Whereas $r=e^{-\varepsilon_{\mathrm{r}}}$ is a global function on $T, \theta=-g_{T}^{*}$ is defined only locally. There is a neighborhood of each point of $T$ except the countably many

Glasgow Math. J. 24 (1983) 101-106. 
critical points of $g_{T}$ in which $r e^{i \theta}$ may be used as the local parameter. Thus we have

$$
D_{G_{\alpha}}(f)=\int_{e^{-\alpha}}^{1}\left(\int_{C_{\alpha}}\left\{\left(\frac{\partial f}{\partial r}\right)^{2}+\frac{1}{r^{2}}\left(\frac{\partial f}{\partial \theta}\right)^{2}\right\} d \theta\right) \rho d \rho
$$

where $C_{\rho}$ is the positively oriented boundary of $\{\rho \in T \mid r(p)<\rho\}$ and $f$ is a suitable function on $G_{\alpha}$. For all but countably many $\rho \in(0,1)$ we know that

$$
\int_{C_{p}} d \theta=2 \pi
$$

(cf. [5, p. 199]). Setting $f=g_{\mathrm{r}}$ in (2) and using (3) to evaluate the right hand side gives $D_{\mathrm{G}_{s}}\left(g_{T}\right)=2 \pi \alpha$. Since $D_{T}\left(\omega_{\alpha}\right)=D_{\mathrm{G}_{\alpha}}\left(\alpha^{-1} g_{T}\right)$, we conclude that (1) holds.

2. We follow the procedure of [3] for forming a Riemann surface by welding subsurfaces of $T$ to a region in the complex plane $\mathbb{C}$. Fix $\nu \in(0,+\infty)$ such that $T \backslash G_{\nu}$ is homeomorphic to a closed disc and does not contain critical points of $g_{T}$. Set $c=2 \pi / \nu$ and fix a number $a \in(1,2)$. Define two sequences

$$
\alpha_{k}=a^{k} \nu, \quad \beta_{k}=2^{k} \nu, \quad k=1,2, \ldots
$$

Note that $G_{\nu} \subset G_{\alpha_{k}} \subset G_{\beta_{k}}$. By the lemma in Section 1 we have

$$
D_{T}\left(\omega_{\alpha_{k}}\right)=c / a^{k}, \quad k=1,2, \ldots
$$

We also remark that $1-\omega_{\beta_{k}} \mid \partial G_{\nu}=\beta_{k}^{-1}\left(g_{T} \mid \partial G_{\nu}\right) \cap 1=\beta_{k}^{-1} \nu \cap 1=2^{-k}$ and in particular,

$$
1-\omega_{\beta_{k}} \mid \partial G_{\nu} \leq \frac{1}{2}, \quad k=1,2, \ldots
$$

We prepare copies $T_{n}, n=1,2, \ldots$, of $T$ and for any $\alpha \in(0,+\infty)$ we let $G_{\alpha}^{n}$ be a copy of $G_{\alpha}$ in $T_{n}$. For $n=1,2, \ldots$ define $X_{n}=G_{\nu}^{n}, W_{n}=G_{\alpha_{n}}^{n}, V_{n}=G_{\beta_{n}}^{n}$. We view $\omega_{\alpha}$ as being defined on $T_{n}$ and set $w_{n}=\omega_{\alpha_{n}}\left|W_{n}, v_{n}=\omega_{\beta_{n}}\right| V_{n}$. We weld the surfaces $V_{n}$ to the region $D=\mathbb{C} \backslash \bigcup_{1}^{\infty}\{|z-3 n| \leq 1\}$ by identifying $\partial V_{n}$ with $\{|z-3 n|=1\}, n=1,2, \ldots$ Denote the resulting Riemann surface by $R$. The functions $w_{n}, v_{n}$ can be viewed as being defined on the subsurfaces $W_{n}, V_{n}$ of $R$. Then according to (4) and (5) we have

and

$$
D_{W_{n}}\left(w_{n}\right)=c / a^{n}
$$

$$
1-v_{n} \mid \partial X_{n} \leq \frac{1}{2}, \quad n=1,2, \ldots
$$

3. Denote the harmonic boundary of $R$ by $\Delta$. Since $\operatorname{dim} H D\left(X_{n} ; \partial X_{n}\right)=1$ we see that $\bar{X}_{n} \cap \Delta$ consists of a single point which we denote by $p_{n}^{*}$. Set $\Delta_{1}=\left\{p_{1}^{*}, p_{2}^{*}, \ldots\right\}$.

Lemma. $\Delta_{1}$ is dense in $\Delta$.

Assume that there is a point $q^{*} \in \Delta \backslash \bar{\Delta}_{1}$. Then we can find a function $u \in H D(R)$ such that $0<u<1, u \mid \bar{\Delta}_{1}=0, u\left(q^{*}\right)=1$. For each $n, u\left(p_{n}^{*}\right)=1-v_{n}\left(p_{n}^{*}\right)=0$ and $u \mid \partial V_{n}<1=$ $1-v_{n} \mid \partial V_{n}$. Thus $u\left|V_{n}<1-v_{n}\right| V_{n}$ and in view of (7) we have $u \mid \partial X_{n} \leq \frac{1}{2}$. Consider 
the region $R_{0}=R \backslash \bigcup_{1}^{\infty} \bar{X}_{n}$. Clearly, $u \mid \partial R_{0} \leq \frac{1}{2}$. Since $R_{0}$ is planar we conclude that $u \mid R_{0} \leq \frac{1}{2}$ and consequently $u \mid R \leq \frac{1}{2}$. This contradicts $u\left(q^{*}\right)=1$ and the assertion follows.

4. Set $W=\bigcup_{1}^{\infty} W_{n}$ and define a function $w$ on $R$ by setting $w|R \backslash W=0, w| W_{n}=$ $w_{n}, n=1,2, \ldots$ Clearly $w \in H B(W ; \partial W)$. By $(6)$ we see that $D_{R}(w)=\sum_{1}^{\infty} D_{W_{n}}\left(w_{n}\right)<+\infty$ and hence $w \in H B D(W ; \partial W)$. Since $w \mid \Delta_{1}=1$, the lemma in Section 3 implies that $w \mid \Delta=1$. Thus $\Delta \subset\left\{p^{*} \in R^{*} \mid w\left(p^{*}\right)>0\right\} \subset \bar{W}$; i.e. $\bar{W}$ is a neighborhood of $\Delta$ in $R^{*}$.

Proposition. The extremization $\mu_{\mathrm{BD}}^{\mathrm{W}}: H B D(W ; \partial W) \rightarrow H B D(R)$ is surjective.

This follows from Theorem 2 of [1] or can be established directly. Let $h$ be any function in $H B D(R)$. Then $w h$ is in the Royden algebra since each factor is and we can set $u=\pi_{\overline{R \backslash W}}(w h)$, the harmonic projection of $w h$ on $W$ (cf. [5, p. 165]). Since $u \in$ $H B D(W ; \partial W)$ and $\mu_{B D}^{W} u|\Delta=u| \Delta=w h|\Delta=h| \Delta$, it follows that $\mu_{B D}^{W} u=h$.

5. Define a measure $\sigma$ on $\Delta$ as follows:

$$
\sigma\left(\Delta \backslash \Delta_{1}\right)=0, \quad \sigma\left(\left\{p_{n}^{*}\right\}\right)=D_{W_{n}}\left(w_{n}\right), \quad n=1,2, \ldots
$$

The space $H D(W ; \partial W)$ is completely determined by $\sigma$.

Proposition. $H D(W ; \partial W) \mid \Delta=L^{2}(\Delta, \sigma)$.

Assume that $u \in H D(W ; \partial W)$. Then $u \mid W_{n}=u\left(p_{n}^{*}\right) w_{n}$ and hence by (8) we see that $+\infty>D_{W}(u)=\sum_{1}^{\infty} D_{W_{n}}(u)=\sum_{1}^{\infty}\left(u\left(p_{n}^{*}\right)\right)^{2} \sigma\left(\left\{p_{n}^{*}\right\}\right)$. Conversely, let $\xi \in L^{2}(\Delta, \sigma)$ and define $\xi_{n}=$ $\xi\left(p_{n}^{*}\right)$. The function $u=\sum_{1}^{\infty} \xi_{n} w_{n}$ is in $H D(W ; \partial W)$ and $u\left(p_{n}^{*}\right)=\xi_{n}, n=1,2, \ldots$ Thus $u \mid \Delta=\xi, \sigma-$ a.e.

6. Recall that the definitions of $W$ and $\sigma$ depend on an arbitrary constant $a \in(1,2)$. Set $W^{a}=W$ and $\sigma^{a}=\sigma$ to express this dependence.

Proposition. The subset $W^{a}$ of $R$ has the property that the deficiency of $\mu_{D}^{W^{a}}$ is infinite.

For the proof set $Z^{a}=\mu_{D}^{W^{a}}\left(H D\left(W^{a} ; \partial W^{a}\right)\right)$. By (8) and (6) it is easily seen that for $a^{\prime}, a^{\prime \prime}$ with $a \leq a^{\prime}<a^{\prime \prime}<2$ the space $L^{2}\left(\Delta, \sigma^{a^{\prime}}\right)$ is properly contained in $L^{2}\left(\Delta, \sigma^{a \prime}\right)$. Since extremization leaves values on $\Delta$ fixed, the proposition in Section 5 gives $Z^{a} \mid \Delta=L^{2}\left(\Delta, \sigma^{a}\right)$ and similarly for $a^{\prime}, a^{\prime \prime}$. We conclude that $Z^{a}$ is contained in $Z^{a^{\prime}}$ and that $Z^{a^{\prime}}, a<a^{\prime}<2$, form a family of properly increasing subspaces of $H D(R)$.

7. We revert to the notations $W$ and $\sigma$. In the remainder of this paper we show that the open set $W$ is contained in a region $\Omega$ such that

$$
H D(\Omega ; \partial \Omega)|\Delta=H D(W ; \partial W)| \Delta .
$$

Actually, in order to make $\partial \Omega$ piecewise analytic it will be necessary to shift our 
considerations to a Riemann surface $R^{\prime}$ obtained from $R$ by removing a point $s_{0}$ from $R$. Since the harmonic boundary of $R^{\prime}$ can be identified with $\Delta$ and $s_{0}$ will not be in $\bar{W}$, the propositions in Sections 4 and 6 will not be affected. The result in (9) implies that the image of $\mu_{D}^{\Omega}$ coincides with the image of $\mu_{D}^{W}$ in $H D\left(R^{\prime}\right)$ and in particular that the image of $\mu_{B D}^{\Omega}$ coincides with the image of $\mu_{B D}^{W}$. Thus our main result will follow from (9) and the two propositions just mentioned.

THEOREM. There exists a Riemann surface $R^{\prime}$ and a subregion $\Omega$ with $\partial \Omega$ piecewise analytic such that $\mu_{B D}^{\Omega}$ is surjective yet the deficiency of $\mu_{D}^{\Omega}$ is infinite.

The region $\Omega$ will consist of the union of $W$, a fixed parametric disc and a collection of thin strips joining the components of $W$ to this disc. Fix a point $p_{0}$ in the subset $D$ of $R$ (cf. Section 2) and let $g_{R}$ be the Green's function for $R$ with pole at $p_{0}$. Choose $\gamma \in(0,+\infty)$ such that the set $B_{\gamma}=\left\{p \in R \mid g_{R}(p)>\gamma\right\}$ is homeomorphic to a disc and $\bar{B}_{\gamma}$ contains no critical points of $g_{R}$. We now fix the arbitrary constant $a \in(1,2)$ that determines $W$ so that none of the critical points of $g_{R}$ lie on $\partial W$.

For each positive integer $n$ let $p_{n}$ be a point in the compact set $\partial W_{n}$ such that $g_{R}\left(p_{n}\right)=\max _{\partial W_{n}} g_{R}$. Then grad $g_{R}$ at $p_{n}$ is perpendicular to the tangent to $\partial W_{n}$ at $p_{n}$. Here we are using the notations $d r, d \theta$ for the polar coordinate differentials of $R$ obtained from $g_{R}$. In terms of the local parameter $r e^{i \theta}$ we can represent $\partial W_{n}$ in a neighborhood of $p_{n}$ in the form $r=f_{n}(\theta), \theta \in I_{n}$, an open interval. The function $\theta$ has a harmonic continuation along any integral curve of grad $g_{R}$ with $\theta$ being constant on the curve. Thus we may choose $\theta_{n} \in I_{n}$ such that the maximal integral curve of grad $g_{R}$ starting at the point $q_{n} \in \partial W_{n}$ with $\theta\left(q_{n}\right)=\theta_{n}, r\left(q_{n}\right)=f_{n}\left(\theta_{n}\right)$ does not terminate at any of the critical points of $g_{R}$. Therefore, this curve intersects $\partial B_{3 \gamma}$ and we denote by $l_{n}$ the portion connecting $q_{n}$ with $\partial B_{3 \gamma}$.

Let $S_{n}$ be a region on which $\theta$ is well-defined and which contains $l_{n}$ but does not contain any critical point of $g_{R}$. Then $r e^{i \theta}$ may be used as the parameter on $S_{n}$. Denote by $\varepsilon_{0}$ the minimum of the positive quantities $\min _{t \in \partial I_{n}}\left|t-\theta_{n}\right|$ and $\inf _{p \in \partial S_{n}}\left|\theta(p)-\theta_{n}\right|$. For any
$\varepsilon \in\left(0, \varepsilon_{0}\right]$ define

$$
U_{n}^{\varepsilon}=W_{n} \cup\left\{p \in R|| \theta(p)-\theta_{n} \mid<\varepsilon \text { and } e^{-3 \gamma}<r(p) \leq f_{n}(\theta)\right\} .
$$

Clearly $U_{n}^{\varepsilon}$ is a region containing $W_{n}$ with $\partial U_{n}^{\varepsilon}$ piecewise analytic and $\bigcap_{0<\varepsilon \leq \varepsilon_{0}} U_{n}^{\varepsilon}=W_{n} \cup \tilde{l}_{n}$, where $\tilde{l}_{n}=l_{n} \backslash \partial B_{3 \gamma}$. Moreover, if $n \neq n^{\prime}$, then the $U_{n}^{\varepsilon}, U_{n^{\prime}}^{\varepsilon^{\prime}}$ constructed here are disjoint.

8. Consider a fixed positive integer $n$. For each integer $m>\varepsilon_{0}^{-1}$ there is a function $u_{m} \in H D\left(U_{n}^{1 / m} ; \partial U_{n}^{1 / m}\right)$ such that $u_{m}\left(p_{n}^{*}\right)=1$. Extend the definition of $w_{n}$ to $R$ by setting $w_{n} \mid R \backslash W_{n}=0$. It is easily seen that for $m^{\prime}>m>\varepsilon_{0}^{-1}, w_{n} \leq u_{m^{\prime}} \leq u_{m}<1$ on $R$. Thus $u=\lim _{m} u_{m}$ exists on $R$,

$$
w_{n} \leq u \leq u_{m} \text { on } R,
$$

$u \mid W_{n}$ is harmonic and $u \mid R \backslash\left(W_{n} \cap \tilde{l}_{n}\right)=0$. By (10) we see that if we set $u\left(p_{n}^{*}\right)=1$, then $u$ is continuous on $W_{n} \cap\left\{p_{n}^{*}\right\}$. Since each point in $\partial W_{n} \backslash\left\{q_{n}\right\}$ is eventually contained in $R \backslash U_{n}^{1 / m}$, (10) implies that $u$ vanishes continuously on $\partial W_{n} \backslash\left\{q_{n}\right\}$. Let $g_{R}\left(\cdot, q_{n}\right)$ be the 
Green's function on $R$ with pole at $q_{n}$. We apply the $H B$-maximum principle (cf. [5, p. 160]) to $w_{n}+\eta g_{R}\left(\cdot, q_{n}\right)-u$ for any $\eta>0$ and let $\eta \rightarrow 0$ to obtain $w_{n} \geq u$ on $W_{n}$. Consequently, $u=w_{n}$ on $R \backslash l_{n}$. Since the sequence $\left\{u_{m}\right\}$ is eventually harmonic at each point of $R \backslash\left(\partial W_{n} \cup l_{n}\right)$, we conclude that

$$
\lim _{m} \operatorname{grad} u_{m}=\operatorname{grad} w_{n} \text { on } R \backslash\left(\partial W_{n} \cup l_{n}\right) .
$$

For $m^{\prime}>m>\varepsilon_{0}^{-1}$, the function $u_{m}-u_{m}$, vanishes on $\left(\overline{R \backslash U^{m}}\right) \cup\left\{p_{n}^{*}\right\}$ and is therefore orthogonal to a harmonic projection on $U_{m}^{1 / n}$ (cf. $[5$, p. 162]). In particular, $D_{R}\left(u_{m}-u_{m}, u_{m}\right)=0$ and consequently

Similarly,

$$
0 \leq D_{R}\left(u_{m^{\prime}}-u_{m}\right)=D_{R}\left(u_{m}\right)-D_{R}\left(u_{m}\right) \text {. }
$$

$$
0 \leq D_{R}\left(w_{n}-u_{m}\right)=D_{R}\left(w_{n}\right)-D_{R}\left(u_{m}\right) .
$$

Thus $\left\{D_{R}\left(u_{m}\right) \mid m>\varepsilon_{0}^{-1}\right\}$ is an increasing sequence bounded above by $D_{R}\left(w_{n}\right)$. Set $d=\lim _{m} D_{R}\left(u_{m}\right)$. Let $m^{\prime} \rightarrow+\infty$ in (12). By (11) and Fatou's lemma we arrive at $0 \leq$ $D_{R}\left(w_{n}-u_{m}\right) \leq d-D_{R}\left(u_{m}\right)$ which, by the triangle inequality, implies that $\lim D_{R}\left(u_{m}\right)=$ $D_{R}\left(w_{n}\right)$. Choose a positive integer $m_{n}$ such that

$$
D_{R}\left(w_{n}\right) / 2 \leq D_{R}\left(u_{m_{n}}\right) \leq D_{R}\left(w_{n}\right)
$$

We repeat this construction for each $n=1,2, \ldots$ Set $Y_{n}=U_{n}^{1 / m_{n}}, y_{n}=u_{m_{n}}$ and $Y=\bigcup_{1}^{\infty} Y_{n}$. Also define a measure $\tau$ on $\Delta$ by setting $\tau\left(\Delta \backslash \Delta_{1}\right)=0, \tau\left(\left\{p_{n}^{*}\right\}\right)=D_{R}\left(y_{n}\right)$. By (14) and (8) we see that $\sigma(A) / 2 \leq \tau(A) \leq \sigma(A)$ for any subset $A$ of $\Delta$. Thus $L^{2}(\Delta, \sigma)=$ $L^{2}(\Delta, \tau)$. On the other hand, by the reasoning of Section 5 we can establish that

It follows that

$$
H D(Y ; \partial Y) \mid \Delta=L^{2}(\Delta, \tau) \text {. }
$$

$$
H D(Y ; \partial Y)|\Delta=H D(W ; \partial W)| \Delta \text {. }
$$

9. Each component $Y_{n}$ of $Y$ intersects the disc $B_{2 \gamma}$ and $\partial Y \cap \partial B_{2 \gamma}$ has precisely one accumulation point $s_{0}$ in $R$. We now regard $R^{\prime}=R \backslash\left\{s_{0}\right\}$ as a Riemann surface in its own right. Let $R^{\prime *}$ be its Royden compactification and denote by $\hat{B}$ the closure of a set $B$ in $R^{\prime *}$. Let $D_{0}$ be a punctured disc centered at $s_{0}$. Then there is a homeomorphism between $R^{\prime *} \backslash \hat{D}_{0}$ and $R^{*} \backslash \bar{D}_{0}$ with harmonic boundary points corresponding to harmonic boundary points (cf. [5, p. 189]). Since the Green's function of $R^{\prime}$ (with pole at a fixed point in $R^{\prime}$ ) is a potential bounded away from 0 in $\hat{D}_{0}$, all the harmonic boundary points of $R^{\prime}$ are contained in $R^{\prime *} \backslash \hat{D}_{0}$. In this sense the harmonic boundary of $R^{\prime}$ can be identified with $\Delta$. Thus (16) continues to hold when $Y$ and $W$ are viewed as subsets of $R^{\prime}$.

Define $\Omega=Y \cup B_{2 \gamma}$. Clearly $\Omega$ is a region with piecewise analytic $\partial \Omega$ in $R^{\prime}$. The final step in our proof is to show that

$$
H D(\Omega ; \partial \Omega)|\Delta=H D(Y ; \partial Y)| \Delta .
$$


Let $v \in H D(Y ; \partial Y)$. Set $u=\pi_{M} v$ (where $M=\widehat{R^{\prime} \backslash \Omega}$ ). Then $u|\Delta=v| \Delta$ and since $v \mid R \backslash \Omega=0$ we have $u \in H D(\Omega ; \partial \Omega)$. To establish the converse let $f \in M\left(R^{\prime}\right)$ such that $f \mid R^{\prime} \backslash B_{\gamma}=1$ and $f \mid B_{3 \gamma / 2}=0$. The support of grad $f$ is compact in $R^{\prime}$ and consequently, for any $u \in H D(\Omega ; \partial \Omega), f u \in M\left(R^{\prime}\right)$. Moreover, $f u=0$ on $\left(R^{\prime} \backslash \Omega\right) \cup B_{3 \gamma / 2}$ which contains $R^{\prime} \backslash Y$. Therefore the function $v=\pi_{N}(f u)$ (where $N=\widehat{R^{\prime} \backslash Y}$ ) belongs to $H D(Y ; \partial Y$ ) and $v|\Delta=(f u)| \Delta=u \mid \Delta$.

In view of (17) and (16) we conclude that (9) holds for $R^{\prime}$. This establishes the theorem.

\section{REFERENCES}

1. M. Nakai, Extremizations and Dirichlet integrals on Riemann surfaces, J. Math. Soc. Japan, 28 (1976), 581-603. 779-782.

2. M. Nakai, Malformed subregions of Riemann surfaces, J. Math. Soc. Japan, 29 (1977),

3. M. Nakai and S. Segawa, Harmonic dimensions related to Dirichlet integrals, J. Math. Soc. Japan, 29 (1977), 107-121.

4. H. L. Royden, Harmonic functions on open Riemann surfaces, Trans. Amer. Math. Soc. 73 (1952), 40-94.

5. L. Sario and M. Nakai, Classification theory of Riemann surfaces (Springer-Verlag, 1970).

Pennsylvania State University,

UnIVERSITY PARK,

PA 16802 ,

U.S.A. 